\title{
$\lg$ E e lgG específicas para antigénios do Aspergillus fumigatus no diagnóstico da aspergilose broncopulmonar alérgica
}

\section{Specific IgE and IgG to Aspergillus fumigatus antigens in the diagnosis of allergic bronchopulmonary aspergillosis}

Data de receção / Received in: 3/9/2020 Data de aceitação / Accepted for publication in: |3/3/202|

Rev Port Imunoalergologia 202I; 29 (4):243-252

Marília Beltrão' (iD, Ana Reis Ferreira ${ }^{2}$ (D), Ana Maria Pereira ${ }^{3}$ (D), Oksana Sokhatska' (iD, Adelina Amorim ${ }^{4}$ (D), Luís Delgado',3,5 (iD

I Serviço de Imunologia Básica e Clínica, Departamento de Patologia, Faculdade de Medicina, Universidade do Porto, Porto, Portugal

${ }^{2}$ Serviço de Imunoalergologia, Centro Hospitalar Vila Nova de Gaia/Espinho, EPE, Vila Nova de Gaia, Portugal

${ }^{3}$ Laboratório de Imunologia, Serviço de Patologia Clínica, Centro Hospitalar e Universitário de São João, Porto, Portugal

${ }^{4}$ Serviço de Pneumologia, Centro Hospitalar e Universitário de São João, Faculdade de Medicina, Universidade do Porto, Porto, Portugal

${ }^{5}$ CINTESIS@RISE - Centro de Investigação em Tecnologias e Serviços de Saúde, Faculdade de Medicina, Universidade do Porto, Porto, Portugal

\section{RESUMO}

Os doseamentos séricos de anticorpos específicos para o Aspergillus fumigatus (A. fumigatus) são úteis para a orientação diagnóstica de aspergilose broncopulmonar alérgica (ABPA). Objetivo: Avaliar a sensibilização aos componentes purificados do $A$. fumigatus por quantificação de anticorpos específicos $\lg E$ e $\lg G$ no soro de doentes com ABPA ou com asma e sensibilização a fungos, usando como comparadores doentes com asma alérgica não sensibilizados a fungos, doentes com fibrose quística (FQ) sem critérios de ABPA e doentes com aspergiloma pulmonar. Métodos: Avaliação transversal de 57 doentes com os diagnósticos de ABPA ( $n=13), F Q(n=9)$, asma sensibilizada a fungos (ASF, $n=10)$, asma alérgica sem sensibilização a fungos $(A A, n=18)$ e aspergiloma $(n=7)$. A determinação das concentrações séricas de $\operatorname{lgE}$ e $\operatorname{lgG}$ específica ( $\lg \mathrm{g}$ e $\operatorname{s} \lg \mathrm{G}$ ) para $A$. fumigatus $(\mathrm{m} 3)$ e cinco dos seus componentes antigénicos recombinantes ( $r$ Asp $f$ ) $r A s p f I$, rAsp $f 2$, rAsp $f 3$, rAsp $f 4$ e rAsp $f 6$ foi realizada por ImmunoCAP. Resultados: A ABPA diferiu significativamente da ASF, pela sensibilização (slgE $\left.>0,10 \mathrm{kU}_{\mathrm{A}} / \mathrm{L}\right)$ ao an- 
tigénio intracelular $r A s p f 6\left(64 \%\right.$ versus $\left.0 \% Q \mathrm{Qui}^{2}=6,2, \mathrm{p}=0,0 \mathrm{I} 3\right)$ e pelo aumento significativo da slgE para o antigénio extracelular $r A s p f I\left(45,3 ; 0,2-33,7 \mathrm{kU}_{\mathrm{A}} / \mathrm{L}\right.$ versus 0,$\left.3 ; 0, \mathrm{I}-0,3 \mathrm{kU} \mathrm{A}_{\mathrm{A}} / \mathrm{L}, \mathrm{P}=0,04 \mathrm{I}\right)$. Os níveis de slgG para $A$. fumigatus foram significativamente diferentes entre os grupos $(p=0,002)$. No entanto, os níveis de slgG para os $r A s p f$ testados não diferiram entre ABPA, ASF, FQ e aspergiloma. Conclusão: $A$ avaliação da resposta lgE ao $A$. fumigatus para alguns dos seus antigénios excretados $(r A s p f l)$ e intracelulares $(r A s p f 6)$ parece útil na distinção serológica da ABPA em relação à ASF, permitindo melhorar a classificação dos doentes. Os níveis séricos de lgG para os rAsp $f$ não distinguem os diferentes grupos de doentes sensibilizados, não melhorando a descriminação diagnóstica obtida utilizando o extrato total do A. fumigatus.

Palavras-chave: Antigénios recombinantes, asma, Aspergillus fumigatus, aspergiloma, aspergilose broncopulmonar alérgica, fibrose quística, IgE, lgG.

(C) 2021 Sociedade Portuguesa de Alergologia e Imunologia Clínica. Publicado por Publicações Ciência \& Vida. Este é um artigo Open Access sob uma licença CC BY-NC-ND (http://creativecommons.org/licenses/by-nc-nd/4.0/).

\section{ABSTRACT}

The serum assays of specific antibodies to Aspergillus fumigatus (A. fumigatus) are useful for the diagnosis of allergic bronchopulmonary aspergillosis (ABPA). Objective: To evaluate the sensitization to purified components of $A$. fumigatus, quantifying specific lgE and $\lg G$ antibodies to its recombinant allergens in the serum of patients with ABPA or with asthma and sensitization to molds, using as comparators patients with allergic asthma not sensitized to molds, patients with cystic fibrosis (CF) without ABPA criteria and patients with pulmonary aspergilloma. Methods: Cross-sectional evaluation of 57 patients diagnosed with ABPA $(n=13), C F(n=9)$, asthma with fungal sensitization (AFS, $n=10)$, allergic asthma without fungal sensitization (AA, $n=18)$ or aspergilloma $(n=7)$. The quantification of serum specific $\lg E$ and $\lg G$ specific (slgE and $s \lg G$ ) to A. fumigatus $(m 3)$ and five of its recombinant antigenic components (rAsp f), rAsp f I, rAsp f 2, rAsp f 3, rAsp f 4 and rAsp f 6 was performed by ImmunoCAP. Results: ABPA significantly differed from ASF in terms of sensitization (slgE> $0.10 \mathrm{kU}_{A} / \mathrm{L}$ ) to its intra-cellular antigens ( $\mathrm{rAsp} f 664 \%$ versus $0 \%$ Qui ${ }^{2}=6.2$, $p=0.013)$ and higher levels of slgE to its extra-cellular antigen $\mathrm{rAsp} f \mathrm{I}\left(45.3 ; 0.2-33.7 \mathrm{kU}_{A} / \mathrm{L}\right.$ versus $0.3 ; 0.1-0.3 \mathrm{k} U_{A} / L$, $p=0.04 I)$. The levels of $s / g G$ for $A$. fumigatus were significantly different between groups $(p=0.002)$. However, the $s / g G$ levels for the rAsp $f$ tested did not differ between ABPA, ASF, FC and aspergilloma. Conclusion: The evaluation of the IgE response to A. fumigatus for some of its excreted ( $\mathrm{rAsp} f \mathrm{I}$ ) and intracellular ( $\mathrm{rAsp} f \mathrm{6}$ ) antigens seems to be useful in the serologic distinction of ABPA and asthmatic patients sensitized to molds. Serum IgG levels for rAsp $f$ do not distinguish between different groups of sensitized patients, and do not improve diagnostic discrimination regarding the total extract of $\mathrm{A}$. fumigatus.

Key-words: Allergic bronchopulmonary aspergillosis, aspergilloma, Aspergillus fumigatus, asthma, cystic fibrosis, lgE, lgG, recombinant antigens.

(c) 202I Sociedade Portuguesa de Alergologia e Imunologia Clínica. Published by Publicações Ciência \& Vida. This is an open access article under the CC BY-NC-ND license (http://creativecommons.org/licenses/by-nc-nd/4.0/). 


\section{INTRODUÇÃO}

U ma percentagem significativa da população atópica encontra-se sensibilizada a fungos e a exposição a alergénios fúngicos em asmáticos sensibilizados tem sido relacionada com exacerbações da doença, necessidades terapêuticas aumentadas e mortalidade por asma ${ }^{1,2}$.A aspergilose broncopulmonar alérgica (ABPA) foi descrita em 1952 por Hinson et al. no Reino Unido, sendo uma doença alérgica respiratória observada em I-2\% dos doentes com asma de longa duração e também em 2-15\% dos doentes com fibrose quística (FQ) ${ }^{3-6}$. Agarwal R. et al. classificou em 2013 a ABPA como sendo uma doença pulmonar imunológica causada por uma hipersensibilidade ao Aspergillus fumigatus, manifestando-se com uma asma mal controlada, infiltrados pulmonares recorrentes e bronquiectasias. $O$ diagnóstico de $A B P A$, segundo os critérios propostos, é estabelecido na evidência de uma lgE total elevada (>1000 IU/ml), lgE específica para A. fumigatus elevada $\left(>0,35 \mathrm{kU}_{\mathrm{A}} / \mathrm{L}\right)$ e dois dos seguintes critérios: presença de precipitinas para $A$. fumigatus, opacidades na radiografia de tórax (fixas ou transitórias), eosinofilia no sangue periférico (> 1000 células/ $\mu \mathrm{l})$, bronquiectasias na tomografia computorizada de alta resolução (TCAR) ${ }^{7}$.

Níveis séricos elevados de anticorpos específicos para o A. fumigatus (slgE e slgG) são considerados um bom marcador diagnóstico de ABPA por permitirem confirmar tanto a sensibilização como o nível de exposição atual do doente ${ }^{4,8}$. Fatores dependentes da exposição ambiental, predisposição genética, defeitos na função mucociliar, bem como patologia pulmonar coexistente, predispõem ao desenvolvimento de $\mathrm{ABPA}^{5,9}$. Alguns doentes podem desenvolver uma síndrome semelhante à $A B P A$, mas originada pela sensibilização a outros fungos, situação reconhecida como micose broncopulmonar alérgica (MBPA). Em casos mais graves, a ABPA pode evoluir para fibrose pulmonar e insuficiência respiratória.

A suspeita clínica de ABPA deve levar a um diagnóstico baseado em dados clínicos, laboratoriais (i.e. IgE específica) e imagiológicos, já que um reconhecimento precoce e tratamento adequado pode prevenir a sua pro- gressão para fibrose pulmonar, $5,10,11$. Uma outra patologia pulmonar que se associa à presença de anticorpos específicos para o A. fumigatus é o aspergiloma pulmonar. Esta patologia surge no seguimento da colonização de uma cavidade pulmonar única com uma bola fúngica de $A$. fumigatus no seu interior, originando um micetoma, geralmente em doentes não imunodeprimidos, não atópicos e com doença pulmonar prévia ${ }^{12,13}$.

Esporos fúngicos são detetáveis no ambiente exterior e interior, sendo cada vez mais reconhecidos como um fator relevante no desenvolvimento da asma alérgica ${ }^{2,5,12}$. Tradicionalmente, o diagnóstico da alergia a fungos baseia-se na história clínica, associada a testes in vivo (e.g. testes cutâneos por picada) e quantificação de IgE específica. Contudo, os extratos totais de diferentes fungos variam muito entre si, pelo que a precisão e fiabilidade dos testes diagnósticos depende da qualidade desses extratos e, pela mesma razão, a correlação entre testes cutâneos e serológicos é limitada na alergia a fungos ${ }^{14}$. Os extratos naturais representam uma mistura indefinida de alergénios e materiais não alergénicos, passando apenas após a sua extração por um processo de remoção de lípidos e resíduos ${ }^{15}$. Devido à reatividade cruzada entre alergénios de diferentes fungos, a reatividade in vivo ou in vitro a extratos totais de $A$. fumigatus nem sempre indicará uma sensibilização genuína a esse fungo ${ }^{16,17}$.

Mais recentemente, componentes alergénicos purificados ou produzidos como proteínas recombinantes têm sido progressivamente introduzidos no diagnóstico das doenças alérgicas, particularmente na avaliação de doentes polissensibilizados ${ }^{18,19}$.

Entre as 23 proteínas alergénicas do $A$. fumigatus já identificadas, é possível atualmente avaliar a reatividade da $\lg \mathrm{E}$ e de $\lg \mathrm{g}$ para cinco desses componentes ( $\mathrm{rAsp} f \mathrm{I}$, $f 2, f 3, f 4$ e $f 6$ ), sendo que vários perfis diferentes de sensibilização têm sido associados à ABPA, com ou sem $\mathrm{FQ}$, e aos doentes com asma e sensibilização a $A$. fumigatus $^{4,20}$. No entanto, a relevância clínica desses perfis de sensibilização é ainda hoje controversa, uma vez que perfis diferentes têm sido descritos em grupos diagnósticos diferentes e em zonas geograficamente distintas ${ }^{21,22}$. 
O objetivo deste estudo foi avaliar a sensibilização aos componentes purificados do A. fumigatus, quantificando anticorpos específicos para os mesmos no soro de doentes com ABPA ou com asma e sensibilização a fungos, usando como comparadores doentes com asma alérgica não sensibilizados a fungos, doentes com FQ sem critérios de ABPA e doentes com aspergiloma pulmonar.

\section{MÉTODOS}

\section{Desenho do estudo}

Avaliação transversal de 57 doentes seguidos em ambulatório num hospital central universitário, com diagnóstico prévio por médico especialista (imunoalergologia e/ou pneumologia) de ABPA (n=13), FQ sem critérios de ABPA (n=9), asma com sensibilização a fungos (ASF, $n=10$ ), asma alérgica sem sensibilização a fungos (AA, $n=18)$ e aspergiloma $(n=7)$.

Treze doentes preenchiam os critérios diagnósticos de Agarwal para ABPA seropositiva ${ }^{7}$. Os 10 doentes com ASF tinham diagnóstico de asma e evidência de lgE positiva $\left(>0,35 \mathrm{kU}_{\mathrm{A}} / \mathrm{I}\right)$ para, pelo menos, um fungo (entre Aspergillus fumigatus, Alternaria alternata, Cladosporium herbarum e Penicillium spp), por testes cutâneos prick e/ou determinação sérica de IgE específica. Os 18 doentes com diagnóstico de AA apresentavam testes cutâneos prick e/ou determinação de IgE específica positiva para, pelo menos, um alergénio inalante, excluindo fungos. A ausência de sensibilização a aeroalergénios fúngicos foi também confirmada por IgE específica negativa $\left(<0,35 \mathrm{kU}_{\mathrm{A}} / \mathrm{L}\right)$ para uma mistura dos alergénios fúngicos referidos $(\mathrm{mxl})$. Nove doentes tinham o diagnóstico de $\mathrm{FQ}$, sem critérios de ABPA, e sete tinham diagnóstico de aspergiloma. As características dos doentes incluídos estão descritas no Quadro I. Todas as amostras séricas analisadas foram obtidas por conveniência aquando de colheitas de sangue realizadas no decurso de avaliação diagnóstica ou de seguimento dos doentes avaliados, pelo que em algumas amostras não foi possível ter volume suficiente para todas as determinações.

\section{Determinação de IgE e IgG específica para antigénios fúngicos}

Foram quantificadas as concentrações séricas de lgE total, IgE e IgG específica para A. fumigatus (m3) e monocomponentes ( $r A s p f)$ por ensaio imunoenzimático fluo-

Quadro I. Características demográficas e quantificação de lgE, IgG, IgE e lgG específicas para o Aspergillus fumigatus (m3), expressos em mediana (min./máx.)

\begin{tabular}{|c|c|c|c|c|c|}
\hline & ABPA & ASF & AA & Aspergiloma & FQ \\
\hline$N$ & 13 & 10 & 18 & 7 & 9 \\
\hline Idade (anos) & $55(10-83)$ & $54(16-77)$ & $40(6-78)$ & $56(36-73)$ & $23(18-37)$ \\
\hline Sexo (F/M) & $7 / 6$ & $8 / 2$ & $13 / 5$ & $2 / 5$ & $3 / 7$ \\
\hline IgE total (kU/l) & $1860(399-27600)$ & $258(28-3167)$ & II0 (I-203I) & $32(4-144)$ & $14(6-158)$ \\
\hline $\begin{array}{l}\text { IgE específica } A \text {. fumigatus } \\
\left(\mathrm{m}^{3} ; \mathrm{kU}_{\mathrm{A}} \mathrm{L}\right)\end{array}$ & $3,4(0, I-74,8)$ & $0,5(0,1-19,6)$ & - & $0, I(0, I-8,9)$ & $*$ \\
\hline IgG total (mg/dL) & $1220(850-3360)$ & $1030(876-1370)$ & II 100 (897-| 480$)$ & $2030(978-3510)$ & $1710(1300-3190)$ \\
\hline $\begin{array}{l}\text { IgG específica } A \text {. fumigatus } \\
\left(\mathrm{m}^{3} ; \mathrm{mg}_{\mathrm{A}} / \mathrm{L}\right)\end{array}$ & $42, I(5,6-135)$ & $27, I(6,2-128)$ & II,I (3,I-22) & II 5,5 (47,6-2535) & $71,0(50,3-73,2)$ \\
\hline
\end{tabular}

* Só um paciente com $10,2 \mathrm{kU} / \mathrm{L}$ os restantes $<0,10 \mathrm{kU} / \mathrm{L}$

ABPA - Aspergilose broncopulmonar alérgica; ASF - Asma com sensibilização a fungos; AA - Asma alérgica sem sensibilização a fungos; FQ - Fibrose quística 
rométrico (CAP-FEIA, Thermo-Fisher ${ }^{\circledR}$ ), seguindo as instruções do fabricante. Resumidamente, o antigénio de interesse, ligado covalentemente a um polímero de celulose tridimensional, reage com a lgE ou lgG específica do soro, previamente diluído, de cada doente. Após a incubação com diluições apropriadas da amostra e da remoção, por lavagem, dos anticorpos não específicos que não se ligaram, é adicionado um imunossoro anti-lgE ou anti-lgG marcado com uma enzima. Depois de uma nova incubação e lavagens adicionais, é adicionado um substrato e a fluorescência no eluído é medida. A resposta em cada amostra é diretamente comparada com calibradores $\lg E\left(\mathrm{kU}_{\mathrm{A}} / \mathrm{L}\right)$ ou $\operatorname{lgG}\left(\mathrm{mg}_{\mathrm{A}} / \mathrm{L}\right)$.

Foram quantificados os anticorpos específicos para uma mistura de extratos totais de fungos ( $\mathrm{mxl}$ - Penicillium chrysogenum, Cladosporium herbarum, Aspergillus fumigatus, Alternaria alternata), para um extrato total purificado de A. fumigatus (m3), bem como para cinco dos seus monocomponentes antigénicos recombinantes intracelulares ( $r A s p f 1, r A s p f 2, r A s p f 3$ ) e excretados ( $r A s p f 4$ e rAsp f 6): rAsp f I (proteína major com $18 \mathrm{KD}$ pertencente à família de mitogilina das citotoxinas), rAsp f 2 (proteína de ligação ao fibrinogénio com 310 resíduos de aminoácidos) e rAsp f 3 (proteína peroxisomal com 18,45KD), rAsp f 4 (proteína intracelular com 30,04KD, cuja função bioquímica não está definida) e rAsp $f 6$ (enzima superóxido dismutase de manganês).

Os níveis de IgE específica para os rAsp $f$ foram considerados como indicando ausência de sensibilização se $<0,10 \mathrm{kU}_{\mathrm{A}} / \mathrm{L}$, o limite de quantificação para o qual o imunoensaio utilizado foi otimizado ${ }^{23}$. A IgG específica para ○ A. fumigatus (m3) foi considerada positiva para níveis $>20 \mathrm{mg}_{\mathrm{A}} / \mathrm{L}^{22}$.

\section{Análise estatística}

A análise descritiva das variáveis é apresentada, exceto quando referido, como mediana e intervalo interquartil (IQR). A significância das diferenças das variáveis contínuas entre grupos ou no mesmo grupo (no caso dos monocomponentes) foi avaliada por testes não paramé- tricos (testes de Mann-Whitney ou de Wilcoxon) e as diferenças de distribuição de variáveis categóricas pelo teste de $Q \mathrm{Qi}^{2}$, com correção de Yates $\left(\mathrm{P}_{\mathrm{C}}\right)$. A associação entre variáveis foi avaliada pela correlação de Spearman. Os dados colhidos foram analisados com o programa SPSS versão 20.0 para Windows.

\section{RESULTADOS}

\section{IgE específica para o Aspergillus fumigatus e monocomponentes recombinantes}

Os níveis de IgE total e específica para $A$. fumigatus (m3) foram mais elevados no grupo com ABPA, de acordo com a definição dos grupos selecionados neste estudo. A IgE específica para o extrato total de $A$. fumigatus (m3) foi positiva $\left(\geq 0,35 \mathrm{kU}_{\mathrm{A}} / \mathrm{L}\right)$ em II de $13(85 \%) \mathrm{com}$ ABPA, 6 de 10 (60\%) do grupo ASF e 2 de 7 (29\%) dos doentes com aspergiloma e I dos 9 de doentes com FQ (Quadro I).

Nestes quatro grupos (sensibilizados a fungos), os doentes com ABPA foram também os que apresentaram globalmente níveis séricos mais elevados de lgE específica para os diferentes rAsp f (Figura I, Quadro 2).

$\mathrm{Na} A B P A$, os níveis de lgE para o extrato total de $A$. fumigatus (m3) correlacionaram-se significativamente com os de cada um dos monocomponentes rAsp $f$ avaliados, verificando-se uma correlação elevada entre os componentes que correspondem a antigénios excreta$\operatorname{dos}(r A s p f I / r A s p f 3, r=0,725, p=0,012)$ e moderada para os componentes intracelulares ( $r A s p f 4$ / $r A s p f 6$, $r=0,626, p=0,039)$. Os níveis de lgE específica para $r A s p$ $f 6\left(4,3 ; 0, I-0,3 \mathrm{kU}_{A} / \mathrm{L}\right)$ foram mais baixos e significativamente inferiores aos dos componentes excretados - rAsp fI $\left(45,3 ; 0,2-33,7 \mathrm{kU}_{\mathrm{A}} / \mathrm{L} ; \mathrm{p}=0,04 \mathrm{I}\right)$ e rAsp f3 $(23,8$; $\left.\mathrm{I}, 0-48, \mathrm{IkU}_{\mathrm{A}} / \mathrm{L} ; \mathrm{P}=0,028\right)$.

O grupo com ABPA diferiu da ASF por um maior número de casos positivos ao rAsp f6 $\left(64 \%\right.$ versus $0 \%$ Qui ${ }^{2}=6,2$, $\left.P_{c}=0,013\right)$ e níveis séricos mais elevados $(p=0,02$; Figura $I)$, verificando-se também uma tendência para maior positi- 


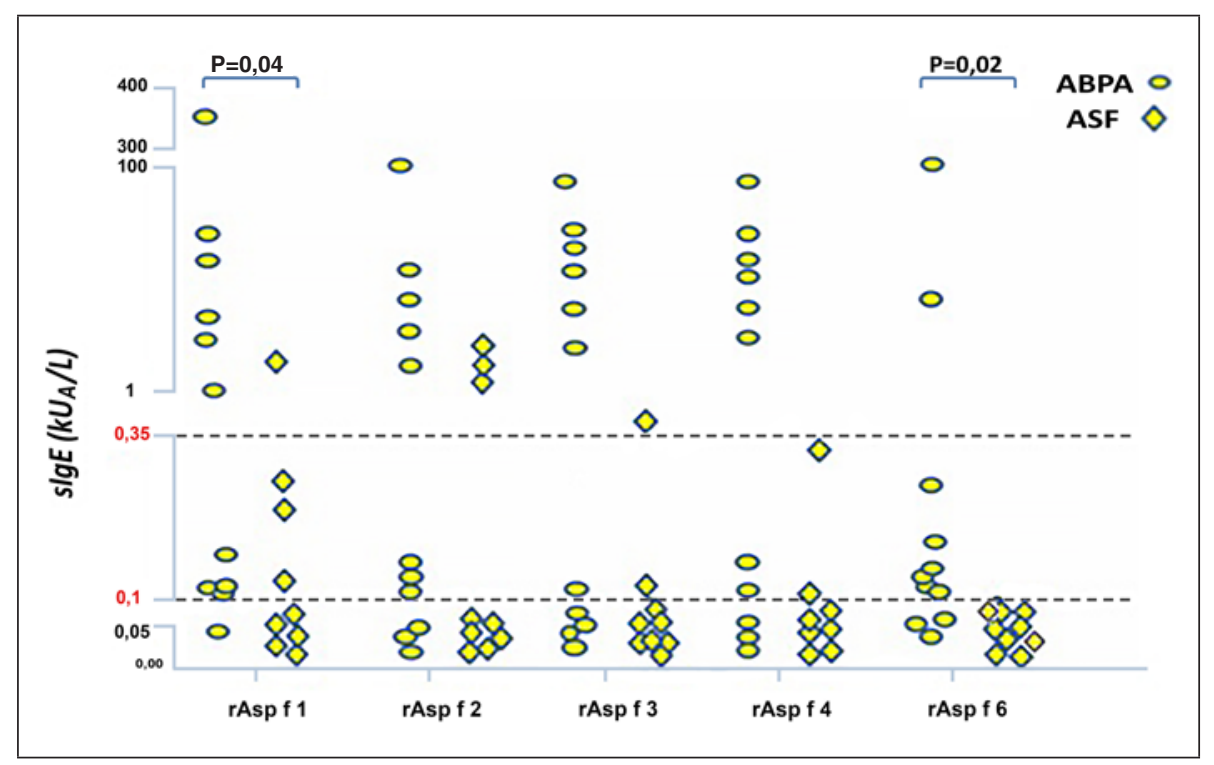

Figura I. Resultados da IgE específica para os diferentes alergénios do Aspergillus fumigatus ( $r$ Asp f, $\mathrm{kUA} / \mathrm{L}$ ) nos doentes com aspergilose broncopulmonar alérgica (ABPA) e asma com sensibilização a fungos (ASF)

Quadro 2. Positividade da IgE específica para os diferentes alergénios do Aspergillus fumigatus ( $r A s p f$ ) nos três grupos sensibilizados e que realizaram todas as determinações. A positividade (\%) está representada a dois níveis $\left(>0,10 \mathrm{e}>0,35 \mathrm{kU}_{\mathrm{A}} / \mathrm{L}\right)$.

\begin{tabular}{|c|c|c|c|c|}
\hline & $k U_{A} / L$ & $\begin{array}{l}\text { ABPA } \\
(n=\mid I)\end{array}$ & $\begin{array}{l}\text { ASF } \\
(n=9)\end{array}$ & $\begin{array}{c}\text { Aspergiloma } \\
\quad(n=6)\end{array}$ \\
\hline \multirow{2}{*}{ rAsp f I } & $>0,10$ & $10(90,9 \%)$ & $4(44,4 \%)$ & $3(50,0 \%)$ \\
\hline & $>0,35$ & $6(54,5 \%)$ & I (II,I \%) & $2(33,3 \%)$ \\
\hline \multirow{2}{*}{$r A s p f 2$} & $>0,10$ & $8(72,7 \%)$ & $3(33,3 \%)$ & I (16,7 \%) \\
\hline & $>0,35$ & $5(45,4 \%)$ & $3(33,3 \%)$ & I (16,7 \%) \\
\hline \multirow{2}{*}{ rAsp f 3} & $>0,10$ & 7 (63,6 \%) & 3 (33,3 \%) & 0 \\
\hline & $>0,35$ & $6(54,5 \%)$ & $2(22,2 \%)$ & 0 \\
\hline \multirow{2}{*}{ rAsp f 4} & $>0,10$ & 8 (72,7 \%) & $2(22,2 \%)$ & I (20\%) \\
\hline & $>0,35$ & $6(54,5 \%)$ & 0 & I (20\%) \\
\hline \multirow{2}{*}{ rAsp f 6} & $>0,10$ & $7(63,6 \%)$ & 0 & 0 \\
\hline & $>0,35$ & $2(18,2 \%)$ & 0 & 0 \\
\hline
\end{tabular}

ABPA - Aspergilose broncopulmonar alérgica; ASF - Asma com sensibilização a fungos.

vidade ao rAsp f $4\left(73 \%\right.$ versus $\left.22 \% \mathrm{Qui}^{2}=3,2, \mathrm{P}_{\mathrm{c}}=0,072\right)$ e rAsp f I ( $91 \%$ versus $44 \%$, Qui $\left.{ }^{2}=3,12, \mathrm{P}_{\mathrm{c}}=0,077\right)$. Quanto à lgE para rAsp f I (o componente mais frequentemente positivo nas nossas amostras), os níveis séricos foram significativamente mais elevados na ABPA do que na ASF $\left(45,3 ; 0,2-33,7 \mathrm{kU}_{A} / L\right.$ versus 0,$\left.3 ; 0, \mathrm{I}-0,3 \mathrm{kU} U_{A} / L, P=0,04 \mathrm{I}\right)$. 


\section{IgG específica para o Aspergillus fumigatus e monocomponentes recombinantes}

Os níveis de lgG para A. fumigatus (m3) (Quadro I) foram significativamente diferentes entre os grupos $(p=0,002)$, também como esperado pela definição das amostras selecionadas. Assim, os níveis mais elevados de lgG para o extrato total de $A$. fumigatus $(\mathrm{m} 3)$ verificaram-se no grupo-controlo com aspergiloma (mediana $115,5 \mathrm{mg} / \mathrm{dL}$, $\mathrm{p}<0,02$ todas as comparações) e os mais baixos na $A A$ (mediana II,I mg/dL, $\mathrm{p}<0,01$ todas as comparações). A lgG específica para $A$. fumigatus $(\mathrm{m} 3)$ apresentou-se também globalmente positiva no grupo com ABPA, ASF e na FQ (Quadro I), não diferindo significativamente entre estes três grupos de doentes.

Quanto aos níveis séricos de lgG para os diferentes componentes rAsp f (Quadro 3), a reatividade ao rAsp f I foi significativamente mais elevada comparativamente com os outros componentes, seguindo-se o rAsp $f 2$, como sendo o segundo antigénio com níveis mais elevados de
IgG específica em qualquer dos grupos com ABPA, ASF, aspergiloma e $\mathrm{FQ}$. No entanto, os níveis de IgG específica para os diferentes $r A s p f$ testados não diferiram significativamente entre os grupos ABPA, ASF, FQ e aspergiloma.

\section{DISCUSSÃO}

Neste estudo transversal de 57 doentes com patologia respiratória que frequentemente se associa a sensibilização ao A. fumigatus podemos verificar que a avaliação da IgE específica para alguns dos seus antigénios excretados $(r A s p f I)$ e intracelulares $(r A s p f 6)$ parece útil na caracterização serológica da ABPA. Assim, a positividade IgE para rAsp f 6 é significativamente superior na ABPA em comparação à da asma alérgica com sensibilização a fungos. Do mesmo modo, os níveis de lgE para rAsp $f$ I apresentam-se significativamente mais elevados na ABPA;

Quadro 3. IgG específica para o extrato total ( $\left.m 3, \mathrm{mg}_{\mathrm{A}} / \mathrm{L}\right)$ e para os diferentes alergénios do Aspergillus fumigatus ( $r$ Asp $f$, $\left.\mathrm{mg}_{\mathrm{A}} / \mathrm{L}\right)$ em doentes com aspergilose broncopulmonar alérgica (ABPA), asma com sensibilização a fungos (ASF), aspergiloma e fibrose quística (FQ), expressos em mediana (min./máx.) do número $(\mathrm{n})$ de amostras quantificadas para todos os componentes.

\begin{tabular}{|c|c|c|c|c|c|c|}
\hline $\begin{array}{c}\text { IgG } \text { mg }_{\mathrm{A}} / \mathbf{L} \\
\text { para: }\end{array}$ & Asp $f(m 3)$ & rAsp f I & rAsp f 2 & $r$ Asp $f 3$ & rAsp f 4 & rAsp f 6 \\
\hline \multirow{3}{*}{ ABPA } & 42,1 & 5,9 & 4,5 & 2,3 & 3,6 & 4,9 \\
\hline & $(5,6-56,8)$ & $(4,2-14,0)$ & $(2,6-6,7)$ & $(0,0-3,9)$ & $(2, I-8,4)$ & $(3,2-7,4)$ \\
\hline & $n=10$ & $n=10$ & $n=9$ & $n=9$ & $n=7$ & $n=10$ \\
\hline \multirow{3}{*}{ ASF } & 22,3 & 5,8 & 5,4 & 3,0 & 3,1 & 3,5 \\
\hline & $(6,2-I \mid 6,0)$ & $(2,5-12,1)$ & $(0,0-10,6)$ & $(0,0-9,6)$ & $(0,0-5,9)$ & $(0,0-8,2)$ \\
\hline & $n=8$ & $n=8$ & $n=8$ & $n=8$ & $n=7$ & $n=7$ \\
\hline \multirow{3}{*}{ Aspergiloma } & 144,0 & 54,9 & 8,9 & 5,5 & 4,7 & 3,0 \\
\hline & $(47,6-2535,0)$ & $(8, I-7 \mid, 6)$ & $(4,2-49,0)$ & $(I, 0-\mid I, 0)$ & $(3,4-5,9)$ & $(1,0-21,8)$ \\
\hline & $n=5$ & $n=5$ & $\mathrm{n}=5$ & $n=5$ & $n=5$ & $n=5$ \\
\hline \multirow{3}{*}{$\mathrm{FQ}$} & 50,3 & 33,2 & 8,8 & 2,7 & 4,6 & 4,9 \\
\hline & $(5,6-73,2)$ & $(10,0-36,8)$ & $(4,7-\mid 4,1)$ & $(2,3-6,5)$ & $(3,5-8,4)$ & $(4,0-10,0)$ \\
\hline & $n=7$ & $n=7$ & $n=7$ & $n=7$ & $n=7$ & $n=7$ \\
\hline
\end{tabular}


nos doentes com ASF, a maioria dos casos com slgE positiva para $r A s p f I$ tinham níveis relativamente baixos (entre 0,II e 0,35kU $/$ L, Quadro 2).

Quanto à sensibilização $\lg G$ para o A. fumigatus (m3), os seus níveis são significativamente mais elevados no aspergiloma e baixos na AA e globalmente positivos e semelhantes nos outros três grupos de doentes, traduzindo provavelmente a exposição frequente a este fungo nas patologias respiratórias incluídas. Apesar de no grupo com ASF os valores de slgG para $A$. fumigatus serem relativamente mais elevados do que no grupo AA (Quadro I), os seus valores medianos $\left(27 \mathrm{mg}_{A} / L\right)$ encontram-se ainda dentro de valores de referência obtidos em diferentes estudos, avaliando, como grupos-controlo, quer dadores de sangue quer doentes respiratórios sem aspergilose ${ }^{22,24}$. Já a lgG específica para os diferentes componentes $r A s p f$, sendo mais elevada para o $r A s p f I$ e rAsp $f 2$, não parece oferecer melhor discriminação diagnóstica que a lgG para o extrato total do A. fumigatus (m3).

Estes resultados estão em consonância com os obtidos num dos estudos iniciais realizados por Crameri $R$ et a ${ }^{25}$, no qual avaliaram a diferente resposta serológica de 60 asmáticos sensibilizados para $A$. fumigatus com ABPA e 40 asmáticos sem ABPA, face aos mesmos alergénios recombinantes. Nos resultados verificaram que os alergénios major rAsp $f$ I e rAsp f 3 são reconhecidos por ambos os grupos com elevada incidência, em contraste com rAsp f 4 e rAsp f6, reconhecidos exclusivamente pelos doentes com ABPA. Em estudos subsequentes ${ }^{4,20}$, os mesmos investigadores avaliaram doentes com FQ com ABPA, um grupo geograficamente mais alargado de doentes com asma e sensibilização a A. fumigatus, bem como controlos normais; os resultados reforçaram a positividade a rAsp $f$ I e rAsp $f$ 3 como marcadores de sensibilização IgE e a positividade a rAsp f 4 e rAsp f 6 mostrou-se relevante no serodiagnóstico da ABPA. Em contraste com estes estudos, Kurup VP et al, avaliando o diagnóstico de ABPA no contexto de $\mathrm{FQ}^{21,26}$, concluem que, apesar de uma resposta IgE um pouco mais elevada para $r A s p f 3$, nenhum dos monocomponentes testados foi capaz de diferenciar a FQ com ABPA de outros doentes com FQ. Já um estudo alargado de casos de ABPA e de asma sensibilizados ao $A$. fumigatus no Japão (306 doentes), recentemente publicado ${ }^{27,28}$, sugere que a IgE específica para rAsp f I (como no presente estudo) e rAsp f 2 efetivamente diferenciam as duas populações, podendo ser considerados como marcadores genuínos de sensibilização a $A$. fumigatus na $A B P A$.

Os resultados descritos em populações de áreas geográficas diferentes e patologias diversas (i.e., com ou sem $\mathrm{FQ}$ ) têm-se mostrado inconsistentes. Uma revisão sistemática recente ${ }^{29}$ concluiu, a partir de 17 estudos e $1|3|$ doentes, que, em doentes com asma, a sensibilidade para o diagnóstico de ABPA é melhor para a combinação de slgE para $r A s p f I$ ou rAsp $f 3$, mas a especificidade associa-se melhor à positividade ao rAsp $f 4$ ou $r A s p f 6$. A resposta imunológica mais específica a estas duas últimas proteínas intracelulares em indivíduos asmáticos com ABPA poderá dever-se ao facto de não se apresentarem como aeroalergénios, em contraste com o que acontece nos asmáticos sensibilizados a fungos, já que na ABPA a colonização das vias aéreas pelo fungo e a subsequente reação imunológica poderá resultar a destruição das estruturas fúngicas e exposição subsequente a estes antigénios intracelulares ${ }^{23}$.

Assim, a avaliação da resposta lgE para monocomponentes do $A$. fumigatus, particularmente para os seus antigénios excretados ( $r A s p$ fl/rAsp f3) versus os intracelulares ( $r A s p f 4 / r A s p f 6$ ), parece útil na caracterização serológica da ABPA. O uso dos alergénios recombinantes permitirá também contornar e distinguir a reatividade cruzada entre alergénios de diferentes fungos (Alternaria alternata, Cladosporium herbarum, Trichophyton rubrum, Malassezia spp.) dos casos especificamente sensibilizados ao Aspergillus fumigatus ("verdadeiras" sensibilizações) ${ }^{23}$. Por outro lado, os níveis séricos de lgG para os rAsp $f$ não distinguem os diferentes grupos de doentes sensibilizados, não melhorando a discriminação diagnóstica da lgG para o extrato total do A. fumigatus (m3).

Este estudo transversal apresenta algumas limitações, como seja amostragem de conveniência (as colheitas de 


\section{IgE E IgG ESPECÍFICAS PARA ANTIGÉNIOS DO ASPERGILLUS FUMIGATUS NO DIAGNÓSTICO DA ASPERGILOSE BRONCOPULMONAR ALÉRGICA / ARTIGO ORIGINAL}

amostras foram realizadas para apoio diagnóstico ou de seguimento e estabelecidas pelo médico especialista assistente), não tendo sido consideradas a fase de evolução ou de tratamento das doenças, aspetos que podem influenciar os níveis de anticorpos séricos específicos. Pelo mesmo motivo, também em alguns casos pontuais não foi possível ter volume suficiente para todas as determinações (ex. $\lg G$ ).

No entanto, salienta-se que a nossa amostragem, comparativamente com as dos outros estudos, agrega uma diversidade de patologias respiratórias associadas ao $A$. fumigatus habitualmente observada em cuidados terciários, com doentes seguidos por equipas multidisciplinares e especializadas. A classificação diagnóstica foi revista por dois dos autores, médicos especialistas na área, por consulta dos registos clínicos, não tendo em conta os resultados da presente investigação dos monocomponentes rAsp f. Utilizámos também neste trabalho um tipo de imunoensaio semiautomático (ImmunoCAPTM) e com reagentes disponíveis comercialmente, cuja calibração, otimização e reprodutibilidade está bem estabelecida em publicações anteriores ${ }^{22}$. Os alergénios recombinantes testados, para IgE e lgG específicas, são também mais estandardizáveis do que os obtidos de extratos nativos, podendo ser preparados de uma forma consistente em grandes volumes $\mathrm{e}$ utilizáveis em equipamentos automatizáveis ${ }^{30}$.

A utilidade da caracterização laboratorial dos componentes alergénicos do $A$. fumigatus deverá ser explorada em estudos longitudinais, envolvendo maior número de doentes, para que seja possível avaliar a sua relevância na deteção de exacerbações e melhor estabelecer critérios de resposta terapêutica numa doença crónica e debilitante como a ABPA.

\section{Financiamento}

Parcialmente suportado pela bolsa da UP Projetos Pluridisciplinares n.130/2010

\section{Conflito de interesses}

Os autores declaram que não existem conflitos de interesses.
ORCID:

Marília Beltrão (ID) 0000-0002-3297-0445

Ana Luísa (iD) 0000-0002-2446-855।

Ana Maria Pereira (DD 0000-0002-4586-9936

Oksana Sokhatska (iD) 0000-0003-3061-8066

Adelina Amorim (ID) 0000-0003-0357-8900

Luís Delgado (iD) 0000-0003-2375-907I

Autor correspondente:

Marília Beltrão

Serviço de Imunologia Básica e Clínica

Departamento de Patologia

Faculdade de Medicina, Universidade do Porto

4200-319 Porto, Portugal

Email: beltraomarilia@gmail.com

\section{REFERÊNCIAS}

I. Knutsen AP, Bush RK, Demain JG, Denning DW, Dixit A, Fairs $\mathrm{A}$, et al. Fungi and allergic lower respiratory tract diseases. J Allergy Clin Immunol 2012;129:280-9I. doi: 10.1016/j.jaci.20II.12.970.

2. Oliveira M, Ribeiro H, Delgado J, Abreu IJA. Seasonal and intradiurnal variation of allergenic fungal spores in urban and rural areas of the North of Portugal. Aerobiologia 2009;25:85-98. doi:10.1034/j.1399-3038.2003.00031.x

3. Khan AN, Jones C, Macdonald S. Bronchopulmonary aspergillosis: a review. Curr Probl Diagn Radiol 2003;32:156-68. doi: 10.1016/ s0363-0188(03)00024-0.

4. Crameri R. Recombinant Aspergillus fumigatus allergens: from the nucleotide sequences to clinical applications. Int Arch Allergy Immunol 1998;|I15:99-II4. doi: 10.1159/000023889.

5. Chaudhary N, Marr KA. Impact of Aspergillus fumigatus in allergic airway diseases. Clin Transl Allergy 20II;1:4. doi: 10.1186/20457022-I-4.

6. Lazarus AA, Thilagar B, McKay SA. Allergic bronchopulmonary aspergillosis. Dis Mon 2008;54:547-64. doi: $10.1016 / j$.dis amonth.2008.05.004.

7. Agarwal R, Chakrabarti A, Shah A, Gupta D, Meis JF, Guleria R, et al; ABPA complicating asthma ISHAM working group. Allergic bronchopulmonary aspergillosis: review of literature and proposal of new diagnostic and classification criteria. Clin Exp Allergy 2013;43:850-73. doi: 10.1III/cea.|2|4I.

8. Agarwal R. Allergic bronchopulmonary aspergillosis. Chest 2009;135:805-26. doi: 10.1378/chest.08-2586.

9. Greenberger PA. Allergic bronchopulmonary aspergillosis. J AIlergy Clin Immunol 2002;1 0:685-92. doi: 10.1067/mai.2002.130I79. 
10. de Oliveira E, Giavina-Bianchi P, Fonseca LA, França AT, Kalil J. Allergic bronchopulmonary aspergillosis' diagnosis remains a challenge. Respir Med 2007;101:2352-7. doi: 10.1016/j. rmed.2007.06.018.

II. Moreira AS, Silva D, Ferreira AR, Delgado L. Antifungal treatment in allergic bronchopulmonary aspergillosis with and without cystic fibrosis: a systematic review. Clin Exp Allergy 2014;44:1210-27. doi: $10.1111 /$ cea. 12333 .

12. Cavaleiro Rufo J, Madureira J, Paciência I, Aguiar L, Pereira C, Silva $\mathrm{D}$, et al. Indoor fungal diversity in primary schools may differently influence allergic sensitization and asthma in children. Pediatr Allergy Immunol 2017;28:332-9. doi: 10.1III/pai.12704.

13. Denning DW, Cadranel J, Beigelman-Aubry C, Ader F, Chakrabarti A, Blot S, et al; European Society for Clinical Microbiology and Infectious Diseases and European Respiratory Society. Chronic pulmonary aspergillosis: rationale and clinical guidelines for diagnosis and management. Eur Respir J 2016;47:45-68. doi: 10.1183/13993003.00583-2015.

14. Simon-Nobbe B, Denk U, Pöll V, Rid R, Breitenbach M. The spectrum of fungal allergy. Int Arch Allergy Immunol 2008;145:58-86. doi: 10.1159/000107578.

15. Curin M, Garib V, Valenta R. Single recombinant and purified major allergens and peptides: How they are made and how they change allergy diagnosis and treatment. Ann Allergy Asthma Immunol 2017;119:201-9. doi: 10.1016/j.anai.2016.11.022.

16. Denning DW, Pashley C, Hartl D, Wardlaw A, Godet C, Del Giacco S, et al. Fungal allergy in asthma-state of the art and research needs. Clin Transl Allergy 2014;4:14. doi: 10.1186/20457022-4-I4.

17. Fukutomi Y, Taniguchi M. Sensitization to fungal allergens: Resolved and unresolved issues. Allergol Int 2015;64:32I-3I. doi: 10.1016/j.alit.2015.05.007.

18. Sastre J. Molecular diagnosis in allergy. Clin Exp Allergy 2010;40):1442-60. doi: I0.1III/j.1365-2222.2010.03585.x.

19. Vieira T, Lopes C, Pereira AM, Araújo L, Moreira A, Delgado L. Microarray based lgE detection in poly-sensitized allergic patients with suspected food allergy - an approach in four clinical cases. Allergol Immunopathol (Madr) 2012;40:172-80. doi: 10.1016/j. aller.2011.03.013.

20. Kurup V, Banerjee B, Hemmann S, Greenberger PA, Blaser K, Crameri RJC, et al. Selected recombinant Aspergillus fumigatus allergens bind specifically to IgE in ABPA. Clin Exp Allergy 2000;30:988-93. doi: 10.1046/j.1365-2222.2000.00837.x.
21. Kurup VP, Knutsen AP, Moss RB, Bansal NK. Specific antibodies to recombinant allergens of Aspergillus fumigatus in cystic fibrosis patients with ABPA. Clin Mol Allergy 2006;4:II. doi: I0.II86/I476-796I-4-II.

22. Page ID, Richardson MD, Denning DW. Comparison of six Aspergillus-specific $\lg G$ assays for the diagnosis of chronic pulmonary aspergillosis (CPA). J Infect 2016;72:240-9. doi: 10.1016/j. jinf.2015.II.003.

23. Matricardi PM, Kleine-Tebbe J, Hoffmann HJ, Valenta R, Hilger C, Hofmaier S, et al. EAACI Molecular Allergology User's Guide. Pediatr Allergy Immunol 2016;27 Suppl 23:I-250. doi: 10.1III/ pai. 12563.

24. Sehgal IS, Choudhary H, Dhooria S, Aggarwal AN, Garg M, Chakrabarti A, et al. Diagnostic cut-off of Aspergillus fumigatus-specific IgG in the diagnosis of chronic pulmonary aspergillosis. Mycoses 2018;61:770-6. doi: 10.1III/myc.128I5.

25. Crameri R, Hemmann S, Ismail C, Menz G, Blaser K. Disease-specific recombinant allergens for the diagnosis of allergic bronchopulmonary aspergillosis. Int Immunol 1998;10:1211-6. doi: 10.1093/intimm/10.8.1211.

26. Knutsen AP, Hutcheson PS, Slavin RG, Kurup VP. IgE antibody to Aspergillus fumigatus recombinant allergens in cystic fibrosis patients with allergic bronchopulmonary aspergillosis. Allergy 2004;59:198-203. doi: 10.1046/j.1398-9995.2003.00310.x.

27. Tanimoto H, Fukutomi Y, Yasueda H, Takeuchi Y, Saito A, Watai $\mathrm{K}$, et al. Molecular-based allergy diagnosis of allergic bronchopulmonary aspergillosis in Aspergillus fumigatus-sensitized Japanese patients. Clin Exp Allergy 2015;45:1790-800. doi: 10.1III/ cea.12590. Erratum in: Clin Exp Allergy 2016;46:38I.

28. Kuwabara K, Hirose M, Kato K, Yokoi T, Shiga M, Kondo R, et al. Serological analysis of sensitization in allergic bronchopulmonary aspergillosis: a study on allergen components and interspecies relationships. J Asthma 2020;57:610-7. doi: 10.1080/ 02770903.2019 .1599387$.

29. Muthu V, Sehgal IS, Dhooria S, Aggarwal AN, Agarwal R. Utility of recombinant Aspergillus fumigatus antigens in the diagnosis of allergic bronchopulmonary aspergillosis: A systematic review and diagnostic test accuracy meta-analysis. Clin Exp Allergy 2018;48:1107-36. doi: $10.1111 /$ cea.13216.

30. Crameri R, Lidholm J, Grönlund H, Stüber D, Blaser K, Menz G. Automated specific IgE assay with recombinant allergens: evaluation of the recombinant Aspergillus fumigatus allergen I in the Pharmacia Cap System. Clin Exp Allergy 1996;26:14II-9. 